\title{
Síndrome de burnout en estudiantes de educación superior tecnológica del campus Tierra Blanca en tiempo de covid-19
}

Burnout syndrome in tech higher education students, from the Tierra Blanca campus, in Covid-19 time

Síndrome de burnout em estudantes do ensino superior tecnológico do campus Tierra Blanca na época de covid-19

Julio Fernando Salazar Gómez

Tecnológico Nacional de México, México j.salazar@itstb.edu.mx http://orcid.org/0000-0003-0597-7163

Erika Dolores Ruiz Tecnológico Nacional de México, México erika@itstb.edu.mx http://orcid.org/0000-003-1089-1284

María de Jesús Valdivia Rivera Tecnológico Nacional de México, México Maria.valdivia@itstb.edu.mx http://orcid.org/0000-0001-5477-9599

Manuel Hernández Cárdenas Tecnológico Nacional de México, México Manuel.hernandez@itstb.edu.mx http://orcid.org/0000-0003-2835-0734 


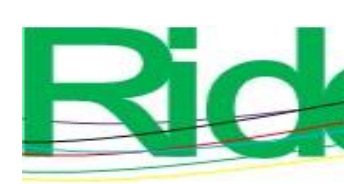

Revista Iberoamericana para la
Investigación y el Desarrollo Educativo
ISSN $2007-7467$

Ibis Rafael Huerta Mora

Tecnológico Nacional de México, México

i.huerta@itstb.edu.mx

http://orcid.org/0000-0001.6815-053X

\section{Resumen}

Esta investigación tuvo como objetivo describir los efectos del síndrome de burnout, en tiempos de pandemia, en estudiantes de educación superior tecnológica. Metodológicamente el estudio tuvo un corte cuantitativo con diseño descriptivo tipo encuesta. Los participantes contestaron el inventario tipo burnout para estudiantes de Maslach, dividido en tres componentes que estudian el agotamiento, el cinismo y la eficacia académica, validado por Shaufeili et al. (2002). La aplicación del instrumento se realizó en el mes de marzo de 2020. Los resultados obtenidos demuestran un agotamiento severo y una eficacia académica positiva; la dimensión cinismo no resultó ser significativa hasta el momento. Las conclusiones se enfocan en sugerir acciones que contribuyan a disminuir el agotamiento en los alumnos, de forma que se pueda mantener la eficacia académica. Asimismo, vale la pena volver a aplicar en el mes de diciembre de 2020 el instrumento para ver la tendencia del burnout en los educandos, ya que existen factores que pueden ser detonantes de estrés, como las clases en línea y la posible acumulación de actividades.

Palabras claves: agotamiento, cinismo, covid-19, eficacia académica, pandemia, síndrome de burnout.

\section{Abstract}

This research aimed to describe the effects of burnout syndrome on students of technological higher education. Methodologically the research had a quantitative cut with descriptive survey-type design, in this context participants answered the burnout inventory for Maslach students divided into 3 components that study exhaustion, cynicism and academic effectiveness validated by Shaufeili et. (2002) the implementation of the instrument took place in March 2020. The results obtained demonstrate severe exhaustion, positive academic effectiveness and the cynic dimension is not significant so far, the conclusions that are drawn in this research are to establish actions that contribute to reducing exhaustion in students so that academic effectiveness can be maintained. As a recommendation it is worth reappliing 


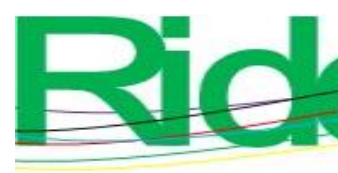

Revista Iberoamericana para la Investigación y el Desarrollo Educativo ISSN $2007-7467$

in december 2020 the instrument to see the trend of burnout in students as there are factors that can be stress triggers to mention online classes and possible accumulation of activities.

Keywords: exhaustion, cynicism, covid-19, academic efficacy, pandemic, burnout syndrome.

\section{Resumo}

Esta pesquisa teve como objetivo descrever os efeitos da Síndrome de Burnout, em tempos de pandemia, em estudantes do ensino superior tecnológico. Metodologicamente, o estudo teve um recorte quantitativo com delineamento descritivo do tipo survey. Os participantes responderam ao inventário de burnout de Maslach para alunos, dividido em três componentes que estudam exaustão, cinismo e eficácia acadêmica, validado por Shaufeili et al. (2002). A aplicação do instrumento foi realizada no mês de março de 2020. Os resultados obtidos mostram grande desgaste e eficácia acadêmica positiva; a dimensão do cinismo não se mostrou significativa até agora. As conclusões se concentram em sugerir ações que ajudem a reduzir o desgaste do aluno, para que a eficácia acadêmica possa ser mantida. Da mesma forma, vale a pena reaplicar o instrumento para verificar a tendência de burnout em alunos em dezembro de 2020, uma vez que existem fatores que podem ser desencadeadores de estresse, como as aulas online e o possível acúmulo de atividades.

Palavras-chave: exaustão, cinismo, covid-19, eficácia acadêmica, pandemia, síndrome de burnout.

Fecha Recepción: Octubre 2020

Fecha Aceptación: Marzo 2021

\section{Introducción}

Actualmente, la población mundial se ve afectada por la pandemia generada por la covid-19, enfermedad que ha cobrado millones de vidas y, debido al continuo confinamiento, ha mermado la salud mental y emocional de todos, lo que se evidencia en altos índices de ansiedad, depresión y estrés. En este contexto, la Organización Mundial de la Salud (OMS) — citada por González y Sandoval (2019) — ha igualado a la salud mental con el bienestar subjetivo, y lo ha caracterizado como un sentimiento de autorrealización de cada una de las capacidades emocionales e intelectuales, aspectos esenciales para enfrentar las tensiones que la vida va presentando. Su contraparte es el agotamiento laboral o síndrome de burnout, 


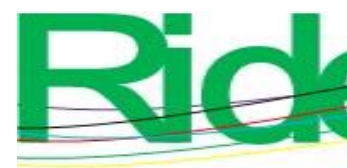

Revista Iberoamericana para la Investigación y el Desarrollo Educativo ISSN 2007 - 7467

concepto que "cobró relevancia como objeto de estudio a partir de la década de 1970, particularmente desde la disciplina de la psicología” (Aguilar, Arvizu y Flores, 2020, p. 4). Este "se caracteriza por una pérdida de ilusión por el trabajo, desgaste psíquico e indolencia y se produce cuando las exigencias del entorno laboral sobrepasan la capacidad de la persona para afrontarlas" (Gallardo, López y Gallardo, 2019, p. 3). Lo anterior suele manifestarse en un cansancio emocional constante y de forma intensa correlacionado con el trabajo, así como en actitudes negativas hacia el entorno y las personas con que se interactúa. Al respecto, Vicente y Gabari (2019) mencionan:

La literatura cientifica describe al bournout como la fase avanzada del estrés profesional, que resulta básicamente de la interacción del trabajador/a con el ambiente laboral, o también producto de las relaciones interpersonales del trabajador y trabajadora con las personas con las que trabaja (p. 456).

Según Camacho (2019), "las teorías vinculadas al estrés han cobrado auge en la gerencia integral del siglo XXI, la cual es estimulada por contextos angustiosos que producen perturbaciones psicológicas y psicosomáticas que son nocivas al ser humano" (p. 42). En otras palabras, una persona que vive constantemente estresada tiende a confrontar ambientes externos que le pueden resultar amenazantes, con lo cual merma su estabilidad al perturbar su equilibrio emocional, lo que recae en su desempeño laboral y en sus relaciones interpersonales. Por ello, León y Monzón (2017) señalan que “el estrés y el sindrome de burnout son los principales riesgos ocupacionales" (p. 322).

En esta línea, Rodríguez, Benavídez, Ornelas y Jurado (2019) mencionan que “el concepto de burnout se ha ampliado a otros ámbitos, pues se ha encontrado que se puede presentar en cualquier circunstancia, es decir, que trasciende a otros contextos" (p. 24), como sucede con el sector educativo. Por este motivo, investigaciones como las de Menghi, Rodríguez y Oñate (2019) se enfocan en señalar que la institución educativa es indispensable para intervenir de forma oportuna ante posibles alteraciones en los alumnos y los docentes.

Bedoya y Vázquez (2019) coinciden en que el estrés es un factor de riesgo que puede ocasionar problemas en la parte cognitiva; de hecho, "la sobrecarga diaria, las tareas diarias y los tiempos reducidos para el esparcimiento en universitarios pueden generar agotamiento emocional" (Castro, Valenzuela, Hinojosa y Piscohe, 2019, pp. 151-152). A esta idea, Usán y Salavera (2019) agregan que "se pueden presentar numerosas situaciones contextuales y personales que pueden afectar de manera notable al alumnado en su proceso de formación” 


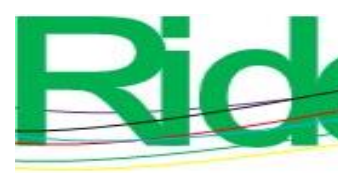

Revista Iberoamericana para la Investigación y el Desarrollo Educativo ISSN 2007-7467

(p. 2). Por ello, Torres (2020) explica que "la reacción que surge ante situaciones sociolaborales y personales [es] indicativa de una condición que sobrepasa a la persona que lo padece" (p. 2).

Reyes, Palomino y Aponte (2019), por su parte, afirman que "el síndrome no solo afecta la calidad de la enseñanza, sino que también perjudica la salud de los docentes" (p. 538). En concordancia con esto, Macías y Báez (2019) encontraron que "76.3 \% de sus participantes presentan la condición de estrés en algún nivel de intensidad” (p. 3). Asimismo, Bresó, Pedraza y Pérez (2019) hallaron en su estudio niveles alarmantes de ansiedad y estrés en cada uno de sus participantes. Aunado a esto, Villamar, Juárez, González y Osnaya (2019) advierten que "el síndrome de burnout se puede manifestar en falta de motivación, interés e irresponsabilidad en las personas" (p. 114).

\section{Método}

El objetivo de la presente investigación fue describir los efectos del síndrome de burnout en estudiantes de educación superior tecnológica. Para ello, se desarrolló un estudio cuantitativo de corte descriptivo y transversal tipo encuesta. La indagación se efectuó en el Tecnológico Nacional de México, campus Tierra Blanca. La población estuvo compuesta por una matrícula de 4700 alumnos que corresponden al sistema escolarizado y semiescolarizado. Se utilizó una muestra probabilística aleatoria simple con un nivel de confianza de $97 \%$ y un margen de error de $3 \%$, lo que arrojó un total de 1023 estudiantes.

El instrumento empleado (validado por Shaufeli, Martínez, Márquez, Salanova y Bakker, 2002) está constituido por 15 ítems agrupados en tres dimensiones: agotamiento (que mide la fatiga), cinismo (que refleja la actitud que toman hacia los estudios) y eficacia académica. Cabe mencionar que si las dimensiones agotamiento y cinismo alcanzan valores elevados y eficacia académica resulta baja, es indicativo de mayor burnout.

Debido a la covid-19, la encuesta se aplicó por medio de classroom para evitar poner en peligro a la población estudiantil. El tratamiento de datos se realizó por medio del software RStudio (versión 1.3). Se determinaron análisis descriptivos (medias y desviación estándar) y se graficó el comportamiento de datos para su mejor interpretación. 


\section{Resultados}

Aplicada la encuesta, se procedió a obtener los resultados en el programa estadístico RStudio (versión 1.3). El test estuvo constituido por 15 ítems en escala de Likert (nunca, ocasionalmente, raramente, casi siempre y siempre) distribuidos en las siguientes dimensiones:

- Agotamiento: 1, 2, 3, 4 y 5.

- Cinismo: 6, 7, 8 у 9 .

- Eficacia académica: 10. 11. 12. 13, 14 y 15.

Los datos indican que no hubo valores pérdidos y que se respetaron en los ítems los valores mínimos (1) y máximos (5), los cuales previamente fueron configurados en la escala de Likert utilizada para el síndrome de bornout. Las preguntas con una varianza más longeva con respecto a la media ubicada en las respuestas casi siempre y siempre fueron las siguientes: 1, 2 y 3, lo que confirma un agotamiento en esta dimensión.

En relación con la dimensión cinismo, la mayoría se encuentra dentro de los factores nunca, ocasionalmente y raramente (preguntas 7, 8 y 9), mientras que solo la pregunta 6 tuvo una varianza de 9.05, orientada a la respuesta siempre y casi siempre con respecto a la media. Interpretados los datos, se confirma que en esta dimensión el tema de cinismo no es significativo.

Por último, en la dimensión eficacia académica, las preguntas 10, 11, 12 y 13 muestran mayor varianza, lo que indica que sus respuestas están dirigidas a casi siempre y siempre con respecto a la media. De hecho, solo las preguntas 14 y 15 cuentan con menos varianza, por lo que se concluye que sí se presenta una eficacia académica (figura 1). 


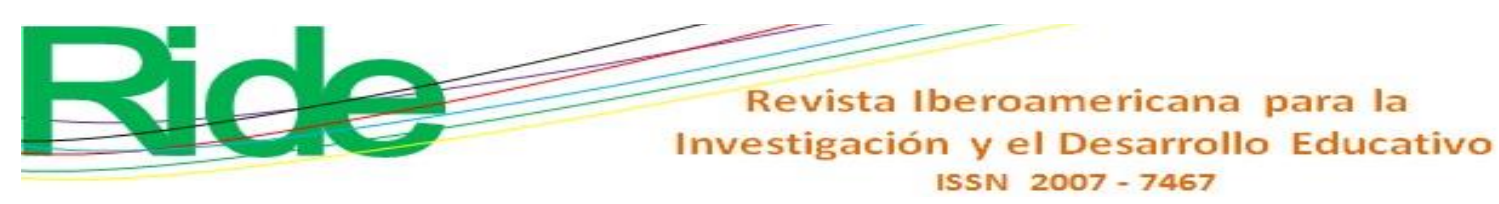

Figura 1. Estadística descriptiva de las dimensiones estudiadas en el inventario de bournot utilizando Rstudio (versión 1.3)

Dimensión agotamiento

$\begin{array}{lccccc} & \begin{array}{c}\text { Pregunta.1 } \\ \text { pregunta.2 }\end{array} & \text { Pregunta. } 3 & \text { Pregunta. } 4 & \text { Pregunta. } 5 \\ \text { nbr. val } & 1.023000 \mathrm{e}+03 & 1.023000 \mathrm{e}+03 & 1.023000 \mathrm{e}+03 & 1.023000 \mathrm{e}+03 & 1.023000 \mathrm{e}+03 \\ \text { nbr. nul1 } & 0.000000 \mathrm{e}+00 & 0.000000 \mathrm{e}+00 & 0.000000 \mathrm{e}+00 & 0.000000 \mathrm{e}+00 & 0.000000 \mathrm{e}+00 \\ \text { nbr. na } & 0.000000 \mathrm{e}+00 & 0.000000 \mathrm{e}+00 & 0.000000 \mathrm{e}+00 & 0.000000 \mathrm{e}+00 & 0.000000 \mathrm{e}+00 \\ \text { min } & 1.000000 \mathrm{e}+00 & 1.000000 \mathrm{e}+00 & 1.000000 \mathrm{e}+00 & 1.000000 \mathrm{e}+00 & 1.000000 \mathrm{e}+00 \\ \text { max } & 5.000000 \mathrm{e}+00 & 5.000000 \mathrm{e}+00 & 5.000000 \mathrm{e}+00 & 5.000000 \mathrm{e}+00 & 5.000000 \mathrm{e}+00 \\ \text { range } & 4.000000 \mathrm{e}+00 & 4.000000 \mathrm{e}+00 & 4.000000 \mathrm{e}+00 & 4.000000 \mathrm{e}+00 & 4.000000 \mathrm{e}+00 \\ \text { sum } & 2.640000 \mathrm{e}+03 & 2.857000 \mathrm{e}+03 & 2.982000 \mathrm{e}+03 & 3.199000 \mathrm{e}+03 & 2.954000 \mathrm{e}+03 \\ \text { median } & 3.000000 \mathrm{e}+00 & 3.000000 \mathrm{e}+00 & 3.000000 \mathrm{e}+00 & 4.000000 \mathrm{e}+00 & 2.000000 \mathrm{e}+00 \\ \text { mean } & 2.580645 \mathrm{e}+00 & 2.792766 \mathrm{e}+00 & 2.914956 \mathrm{e}+00 & 3.127077 \mathrm{e}+00 & 2.887586 \mathrm{e}+00 \\ \text { SE. mean } & 3.085101 \mathrm{e}-02 & 3.651560 \mathrm{e}-02 & 3.913938 \mathrm{e}-02 & 4.160194 \mathrm{e}-02 & 3.205188 \mathrm{e}-02 \\ \text { CI. mean. } 0.95 & 6.053856 \mathrm{e}-02 & 7.165413 \mathrm{e}-02 & 7.680273 \mathrm{e}-02 & 8.163499 \mathrm{e}-02 & 6.289502 \mathrm{e}-02 \\ \text { var } & 9.736759 \mathrm{e}-01 & 1.364057 \mathrm{e}+00 & 1.567124 \mathrm{e}+00 & 1.770528 \mathrm{e}+00 & 1.050951 \mathrm{e}+00 \\ \text { std. dev } & 9.867502 \mathrm{e}-01 & 1.167929 \mathrm{e}+00 & 1.251848 \mathrm{e}+00 & 1.330612 \mathrm{e}+00 & 1.025159 \mathrm{e}+00 \\ \text { coef. var } & 3.823657 \mathrm{e}-01 & 4.181977 \mathrm{e}-01 & 4.294570 \mathrm{e}-01 & 4.255130 \mathrm{e}-01 & 3.550230 \mathrm{e}-01\end{array}$

Dimensión cinismo

Dimensión eficacia académica

\begin{tabular}{|c|c|}
\hline & gunta.10 pregunta.11 Pregunta.12 Pregunta.13 Pregunta.14 Pregunta. \\
\hline or. val & $1.023000 \mathrm{e}+031.023000 \mathrm{e}+031.023000 \mathrm{e}+031.023000 \mathrm{e}+031.023000 \mathrm{e}+031.023000 \mathrm{et}$ \\
\hline nbr. null & $0.000000 \mathrm{e}+00 \quad 0.000000 \mathrm{e}+00 \quad 0.000000 \mathrm{e}+00 \quad 0.000000 \mathrm{e}+00 \quad 0.000000 \mathrm{e}+00 \quad 0.000000 \mathrm{e}+00$ \\
\hline or. na & $00 \mathrm{e}+00 \quad 0.000000 \mathrm{e}+00 \quad 0.000000 \mathrm{e}+000.00$ \\
\hline & $1.000000 \mathrm{e}+001.000000 \mathrm{e}+001.000000 \mathrm{e}+001.000000 \mathrm{e}+001.0$ \\
\hline & $5.000000 \mathrm{e}+005.000000 \mathrm{e}+005.000000 \mathrm{e}+005.0000000 \mathrm{e}+005.00$ \\
\hline nge & $00 \mathrm{e}+004.000000 \mathrm{e}+004.000000 \mathrm{e}+004.000000 \mathrm{e}+004.000000 \mathrm{e}+004.000000 \mathrm{e}+00$ \\
\hline & $2.615000 \mathrm{e}+033.380000 \mathrm{e}+03 \quad 4.156000 \mathrm{e}+03 \quad 3.305000 \mathrm{e}+03 \quad 3.081000 \mathrm{e}+03 \quad 3.970000 \mathrm{e}+03$ \\
\hline dian & $4.000000 \mathrm{e}+005.000000 \mathrm{e}+003.000000 \mathrm{e}+003.0$ \\
\hline an & $3.304008 \mathrm{e}+004.0$ \\
\hline mean & $77 e-024.765611$ e-02 3.089592e-02 4.580481e-02 4.45 \\
\hline mean. 0.95 & 5.84 \\
\hline & 09.7 \\
\hline & e+00 9.8 \\
\hline var & 14.7 \\
\hline
\end{tabular}

\section{Fuente: Elaboración propia}

Se muestra que $64 \%$ de los participantes se sienten emocionalmente agotados por sus estudios, $43 \%$ se sienten agotados al final del día en sus quehaceres universitarios, $41 \%$ se sienten cansados cuando despiertan en la mañana y tienen que enfrentar otro día en la universidad, mientras que para $30 \%$ estudiar o asistir a una clase resulta realmente estresante. Finalmente, solo $7 \%$ considera sentirse agotado por sus estudios. Sin embargo, al analizar los ítems que califican la dimensión agotamiento se encuentra una tendencia muy alta hacia un agotamiento calificado como de intensidad elevada (figura 2). 
Figura 2. Dimensión agotamiento



Fuente: Elaboración propia

Con respecto a la dimesión cinismo, los resultados muestran que $87 \%$ de los estudiantes manifiestan sentir desinterés por sus estudios, $29 \%$ carecen de entusiasmo para estudiar, $6 \%$ considera hacer siempre comentarios burlones sobre la utilidad de lo que estudia, y $50 \%$ lo hace raras veces. Asimismo, $5 \%$ siempre tiene dudas sobre la importancia de sus estudios, mientras que $38 \%$ manifiesta hacerlo raras veces (figura 3). En esta dinámica, la dimensión cinismo se puede considerar como de intensidad baja. 
Figura 3. Dimensión cinismo

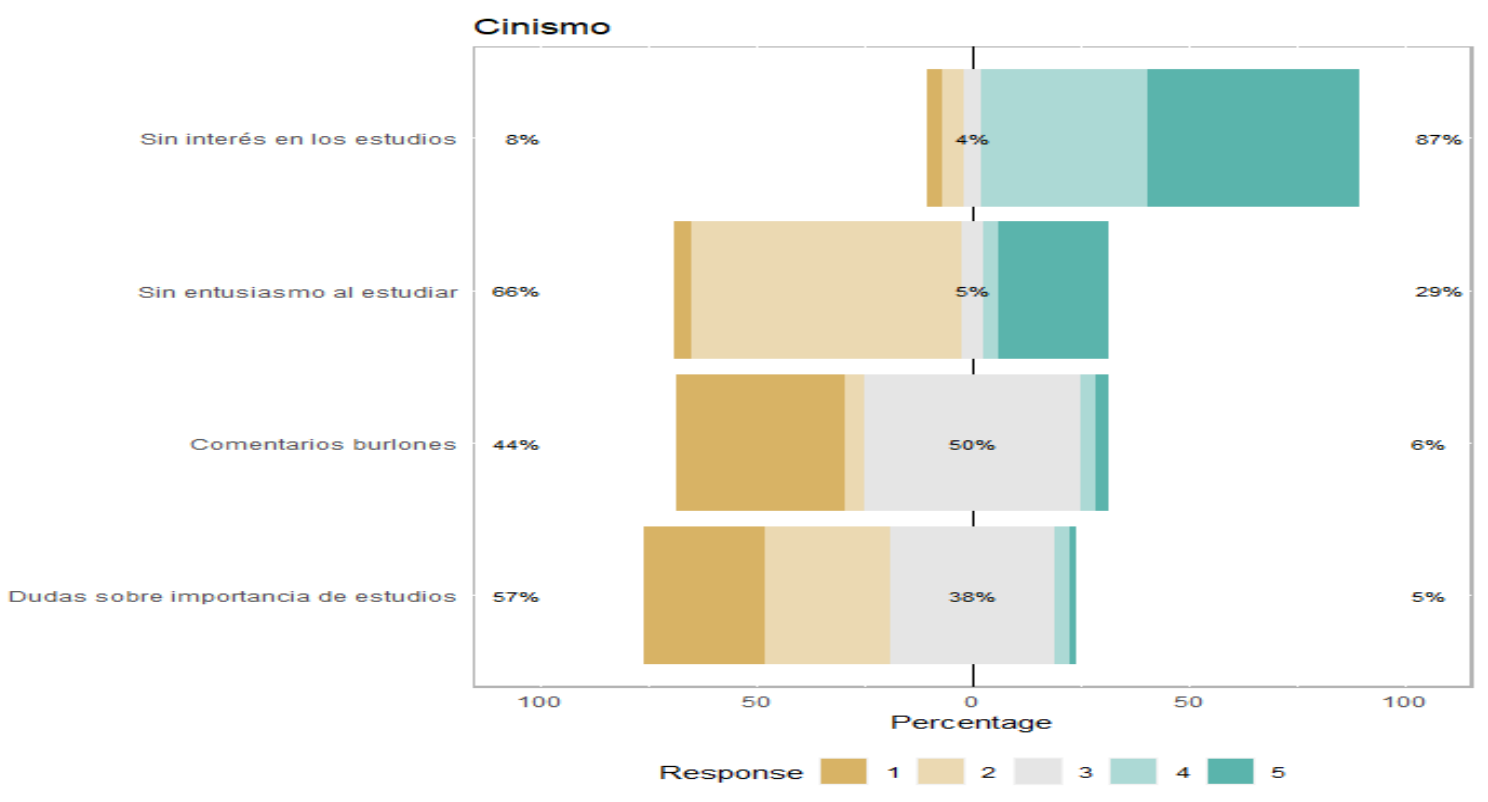

Fuente: Elaboración propia

Por último, en la dimensión eficacia académica, se halló que $62 \%$ de los alumnos pueden resolver eficazmente los problemas que surgen en sus estudios, $58 \%$ cree que puede hacer una contribuación efectiva en las clases a las que asiste, $51 \%$ se considera un buen estudiante, $38 \%$ se siente entusiasmado cuando alcanza las metas, y $29 \%$ ha aprendido cosas interesantes a lo largo de sus estudios; no obstante, $41 \%$ dice que raras veces ha aprendido algo interesante y $6 \%$ menciona que durante la clase se siente seguro de ser competente, aunque en este ítems, $64 \%$ raras veces se siente seguro, por lo que esta dimensión se puede calificar como de intensidad elevada (figura 4). 

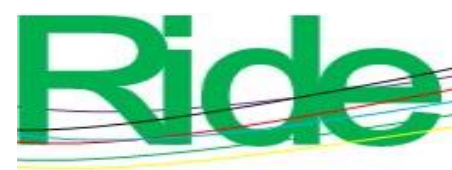

Revista Iberoamericana para la Investigación y el Desarrollo Educativo ISSN 2007 - 7467

Por lo tanto, en relación con la pregunta de investigación planteada (es decir, ¿cómo afecta académicamente a los estudiantes de educación superior tecnológica el síndrome de burnout en esta época de pandemia?), se puede concluir que hasta el momento (marzo de 2020) ha sido una afectación negativa significativa que ha generado un agotamiento excesivo en los alumnos ocasionado por la sobrecarga de actividades y el cambio de ambiente (en línea) para desarrollar las actividades; por ello, es preponderante establecer acciones que contribuyan a disminuir este factor en los alumnos.

\section{Futuras líneas de acción}

Con base en los resultados obtenidos es pertinente continuar con la investigación dado que los tiempos que ha requerido el confinamiento cada día son más extensos en México, por lo que las condiciones ya han cambiado en gran medida. Para futuros proyectos investigativos es recomendable poder analizar como afecta el burnout a las mujeres dadas las condiciones fisiológicas y emocionales que influyen en el género y poder contrastar a ambos sexos bajo el mismo fenómeno. Los resultados pueden ser útiles para establecer estrategias que coadyuven en el programa nacional de tutorías. Por último, es importante estudiar la plantilla docente para comprobar cuales son las afectaciones que el estrés esta causando en ellos y poder encontrar un equilibro en ambos contextos, es decir, estudiantes-docentes y que el proceso de enseñanza-aprendizaje sea realmente significativo.

\section{Agradecimientos}

Se agradece al Tecnológico Nacional de México, campus Tierra Blanca por todas las facilidades otorgadas en el proceso de investigación, desde la autorización, seguimiento del mismo, así como del financiamiento correspondiente. 


\section{Referencias}

Aguilar, J. I., Arvizu, R. y Flores, A. E. (2020). Síndrome de burnout en estudiantes de nivel superior. Análisis comparativo entre estudiantes agrupados por programa educativo dentro del Instituto Tecnológico Superior de Ciudad Constitución. Revista Iberoamericana de las Ciencias de la Salud, 9(17), 1-24. Doi: https://doi.org/10.23913/rics.v9i17.82

Bedoya, E. Y. y Vázquez, D. A. (2019). Estrés y funcionamiento cognitivo en universitarios. Revista Chilena Neuropsicol, 14(1), 23-29. Recuperado de https://repository.ucc.edu.co/bitstream/20.500.12494/17253/1/2019_estres_funciona miento.pdf

Bresó, E., Pedraza, L. y Pérez, K. (2019). Síndrome de burnout y ansiedad en médicos de la ciudad de Santa Marta. Duazary, 16(2), 259-269. Doi: https://doi.org/10.21676/2389783X.2958

Camacho, C. L. (2019). Una mirada desde el síndrome de burnout hacia la gerencia integral en el siglo XXI. Revista Scientific, 4(13), 40-59. Doi: https://doi.org/10.29394/Scientific.issn.2542-2987.2019.4.13.2.40-59

Castro, Y., Valenzuela, O., Hinojosa, M. y Piscohe, C. (2019). Agotamiento emocional en estudiantes de Odontología de la Universidad Nacional Mayor de San Marcos. Revista Habanera de Ciencias Médicas, 18(1), 150-163. Recuperado de http://scielo.sld.cu/scielo.php?script=sci_arttext\&pid=S1729$519 X 2019000100150 \& \operatorname{lng}=\mathrm{es} \& \operatorname{lng}=\mathrm{es}$

Gallardo, J. A., López, F. y Gallardo, P. (2019). Análisis del síndrome de burnout en docentes de educación infantil, primaria y secundaria para su prevención y tratamiento. Revista Electrónica Educare, 23(2), 1-20. Doi: http://dx.doi.org/10.15359/ree.23-2.17

González, R. y Sandoval, J. E. (2019). Manual de psiquiatría. La Habana: ECIMED.

Guillen, A. M., Flores, E., Díaz, J. A., Garza, U., López, R. E., Aguilar, D., Muñoz, J. F. y Rentería, L. (2018). Incremento de síndrome de burnout en estudiantes de Medicina tras su primer mes de rotación clínica. Educación Médica, 20(6), 376-379. Doi: https://doi.org/10.1016/j.edumed.2018.09.003

León, J. J. y Monzón, R. J. (2017). Bienestar psicológico y síndrome de burnout en docentes de las instituciones educativas de jornada escolar completa de dos distritos de la 
provincia de Cajamarca-2017. Revista Perspectiva, 20(3), 320-326. Doi: https://doi.org/10.33198/rp.v20i3.00044

Macías, S. y Báez, Y. (2019). Impacto del síndrome de Burnout en el sector industrial de Baja California. Revista en Ingeniería y Tecnología, 13(2), 1-5. Recuperado de http://editorial-uaie.uaz.edu.mx/index.php/difu100cia/article/view/297/0

Menghi, M. S., Rodríguez, L. M. y Oñate M. E. (2019). Diferencias en los valores de las dimensiones del burnout en educadores con y sin síntomas o problemas de salud. $\begin{array}{lllll}\text { Propósitos } \quad y \quad \text { Representaciones, } & \text { 7(3), 179-197. Doi: }\end{array}$ http://dx.doi.org/10.20511/pyr2019.v7n3.338

Reyes, F. A., Palomino, C. y Aponte, N. W. (2019). Síndrome de desgaste profesional, índice de masa corporal y otros factores asociados con la labor de profesores de educación física de Ibagué, Colombia. Biomédica, 39(3), 537-546. Doi: https://doi.org/10.7705/biomedica.4282

Rodríguez, J. M., Benavídez, E. V., Ornelas, M. y Jurado, P. J. (2019). El burnout académico percibido en universitarios; comparaciones por género. Formación Universitaria, 12(5), 23-30. Doi: http://dx.doi.org/10.4067/S0718-50062019000500023

Shaufeli, W. B., Martínez, I., Márquez, A., Salanova, M. and Bakker, A. (2002) Burnout and Engagement in University Students: A Cross-national Study. Journal of CrossCultural Psychology, 33(5), 464-481. Doi: https://doi.org/10.1177/0022022102033005003

Suárez, Y., Caballero, C., Palacio, J. y Abello, R. (2019). Cambios del burnout académico, engagement y la salud mental durante un semestre de estudio. Duazary, 16(1), 23-37. Doi: https://doi.org/10.21676/2389783X.2530

Torres, E. (2020). Habilidades intrapersonales y su relación con el burnout en docentes de educación básica en León, México. Revista de Estudios y Experiencias en Educación, 19(39), 163-179. Doi: http://dx.doi.org/10.21703/rexe.20201939torres9

Usán, P. y Salavera, C. (2019). Relación entre orientaciones intrínsecas y burnout académico en estudiantes. Psicología Escolar y Educacional, 23(1), 1-40. Doi: https://doi.org/10.1590/2175-35392019018061

Vicente, M. I. y Gabari, M. I. (2019). Niveles de bournout en docentes de secundaria, un estudio descriptivo analítico. Revista Infad de Psicología, 2(1), 455-464. Doi: https://doi.org/10.17060/ijodaep.2019.n1.v2.1485 
Villamar, D., Juárez, A., González, I. G. y Osnaya, M. (2019). Factores psicosociales y síndrome de Burnout en académicos de una universidad pública de México. Propósitos y Representaciones, 7(3), 111-140. Doi: http://dx.doi.org/10.20511/pyr2019.v7n3.360

\begin{tabular}{|l|l|}
\hline Rol de Contribución & Autor (es) \\
\hline Conceptualización & Erika Dolores Ruiz \\
\hline Metodología & Erika Dolores Ruiz \\
\hline Software & Julio Fernnado Salazar Gómez \\
\hline Validación & Manuel Hernández Cárdenas \\
\hline Análisis Formal & Ibis Rafael Huerta Mora \\
\hline Investigación & Erika Dolores Ruiz \\
\hline Recursos & María de Jesús Valdivia Rivera \\
\hline Curación de datos & Julio Fernando Salazar Gómez \\
\hline $\begin{array}{l}\text { Escritura - Preparación del } \\
\text { borrador original }\end{array}$ & Erika Dolores Ruiz \\
\hline $\begin{array}{l}\text { Escritura - Revisión y } \\
\text { edición }\end{array}$ & Julio Fernnado Salazar Gómez \\
\hline Visualización & María de Jesús Valdivia Rivera \\
\hline Supervisión & Erika Dolores Ruiz \\
\hline Administración de Proyectos & Manuel Hernández Cárdenas \\
\hline Adquisición de fondos & Huerta Mora \\
\hline
\end{tabular}

